Markus Beckmann

\title{
Governance durch politische Selbstbindung - Wider die Ausbeutungsspiele afrikanischer Eliten
}

\author{
Problemstellung
}

Nicht erst seit den Vorbereitungen zum G8-Gipfel in Heiligendamm steht der afrikanische Kontinent wieder verstärkt im Blickpunkt der Weltöffentlichkeit. Besondere Aufmerksamkeit wird zurzeit der »Neuen Partnerschaft für die Entwicklung Afrikas (NePAD) « zuteil. Die Beurteilungen der NePAD-Initiative fallen jedoch weit auseinander. Befürworter begrüßen die Initiative als einen entscheidenden Schritt zur Förderung von Demokratie, guter Regierungsführung und nachhaltiger Entwicklung in Afrika. Kritiker bezweifeln dagegen, dass NePAD diese Erwartungen erfüllen wird. In ihren Augen stellt NePAD lediglich einen weiteren, wenn auch geschickten, Versuch, dar, westliche Geberländer zur Zahlung zusätzlicher Entwicklungsgelder zu bewegen - die dann letztlich wieder in den Taschen der herrschenden Eliten versickern würden. Angesichts dieser widersprüchlichen Bewertungen bleibt die Frage strittig, ob - und vor allem: wie - Politik, Wirtschaft und Zivilgesellschaft das NePAD-Programm in Zukunft unterstützen sollen.

Der vorliegende Beitrag zeigt auf, wie der Ansatz der Wirtschaftsethik sozialer Dilemmata $^{1}$ in dieser Situation einen konstruktiven Verständigungsbeitrag leisten

1 Die Wirtschaftsethik wird hier dezidiert als ökonomische Ethik aufgefasst. Wirtschaftsethik wählt in diesem Sinne eine kategorial andere Perspektive als beispielsweise die Medizinethik oder die Wissenschaftsethik. Diese »Bereichs«-Ethiken werden ontologisch von ihrem Gegenstand her konstituiert: als Angewandte Ethik für den Bereich Medizin oder den Bereich Wissenschaft. Der hier verwendete wirtschaftsethische Ansatz konstituiert sich dagegen nicht ontologisch - als »business ethics « -, sondern methodologisch - als »economic ethics «: Wirtschaftsethik ist ökonomische Anreizethik. Verstanden als ein prononciert sozialwissenschaftlicher Ansatz, bietet die Wirtschaftsethik sozialer Dilemmata ein methodisches Instrumentarium, um normative Fragen des gesellschaftlichen Zusammenlebens einer positiven Rational-Choice-Analyse zugänglich zu machen. Vgl. hierzu grundlegend Karl Homann / Ingo Pies, »Wirtschaftsethik in der Moderne. Zur ökonomischen Theorie der Moral «, in: Ethik und Sozialwissenschaften, 1/1994, S. 3-12; Ingo Pies, »Wirtschaftsethik als ökonomische Theorie der Moral. Zur fundamentalen Bedeutung der Anreizanalyse für ein modernes Ethikparadigma «, in: Wulf Gaertner (Hrsg.): Wirtschaftsethische Perspektiven V. Methodische Ansätze, Probleme der Steuer- und Verteilungsgerechtigkeit, Ordnungsfragen, Berlin 2000, S. 1133; Andreas Suchanek, Ökonomische Ethik, Tübingen 2001 sowie Ingo Pies / Markus Sardison, Wirtschaftsethik, in: Knoepffler, Nikolaus, Peter Kunzmann, Ingo Pies und Anne Siegetsleitner (Hrsg.): Einführung in die Angewandte Ethik, Freiburg 2006, S. 267-298. 
kann. Ziel ist es, zunächst das eigentliche Innovationsmoment NePADs genauer zu bestimmen und sodann jene Bedingungen kritisch zu beleuchten, unter denen dieses Potential auch tatsächlich zur Geltung gebracht werden kann. Gleichzeitig bietet der Fall des APRM ein instruktives Beispiel, um die Logik politischer Selbstbindung in transnationalen und innerstaatlichen Reformprozessen systematisch zu reflektieren. Der Aufsatz geht hierzu in drei Schritten vor. Der erste Abschnitt skizziert überblicksartig Entstehung und Inhalt der NePAD-Initiative und arbeitet heraus, in welchen Punkten sich NePAD von früheren afrikanischen Entwicklungsprogrammen grundlegend unterscheidet. Die entscheidende Neuerung, so die These, liegt im so genannten African Peer Review Mechanism (APRM), einem auf Wechselseitigkeit angelegten Informations- und Kontrollinstrument.

Der zweite Abschnitt erläutert die Bedeutung das African Peer Review Mechanism aus einer dezidiert wirtschaftsethischen Perspektive. Auch hier kann die These vorab formuliert werden: Die Unterwicklung afrikanischer Staaten lässt sich als ein Ausbeutungsspiel rekonstruieren, das den Charakter einer sozialen Falle aufweist. In diesem sozialen Dilemma bleiben alle Beteiligten unter ihren Möglichkeiten, weil an sich produktive Investitionen aufgrund drohender Ausbeutung und fehlender Erwartungssicherheit unterbleiben. Um hier Erwartungssicherheit zu ermöglichen, bedarf es einer politischen Selbstbindung der herrschenden Elite. Aufgrund schwacher Staatlichkeit ist eine solche Selbstbindung innerhalb der afrikanischen Einzelstaaten jedoch kaum glaubwürdig. Der African Peer Review Mechanism kann in diesem Sinne als ein Versuch interpretiert werden, Glaubwürdigkeit gleichsam von außen zu importieren.

Der dritte Abschnitt richtet den Blick auf die tatsächliche Entwicklung des APRM. Aufbauend auf der zuvor entwickelten Kritik identifiziert der Beitrag Stärken und Schwächen des APRM und diskutiert die Bedeutung der Integrität des APRM-Verfahrens, die Einbindung der Zivilgesellschaft, die Rolle externer (wirtschaftlicher) Unterstützung sowie die Frage nach der Inklusivität des APRM-Prozesses. Der Beitrag endet mit einem kurzen Fazit.

\section{New Partnership for Africa's Development (NePAD)}

(1) Im September 2000 verständigten sich die Mitgliedstaaten der Vereinten Nationen mit den Millennium Development Goals auf das Ziel, die extreme Armut und den Hunger weltweit - gemessen an den Zahlen von 1990 - bis zum Jahr $2015 \mathrm{zu}$ halbieren. Etwa zeitgleich entstanden mit dem Millennium Africa Plan des südafrikanischen Präsidenten Thabo Mbeki, dem OMEGA Plan des senegalesischen Präsidenten Abdoulaye Wade sowie dem Compact for African Recovery der UN Economic Commission for Africa drei unabhängige Vorschläge für eigenständige Entwicklungsinitiativen in Afrika. ${ }^{2}$ Auf einer gemeinsamen Konferenz im Mai 2001 fassten die afrikanischen Wirtschafts- und Finanzminister den Entschluss, diese un-

2 Alex de Waal, »What's new in the >New Partnership for Africa's Development ?? «, in: International Affairs (3) 2002; S. 463-475, hier S. 466. 
terschiedlichen Ansätze zu einer einzelnen Initiative zusammenzuführen. Im gleichen Jahr initiierten Südafrika und Senegal gemeinsam mit den Präsidenten Algeriens, Nigerias und Ägyptens den gemeinsamen Plan einer New Partnership for Africa's Development. Diesen ursprünglich fünf Gründungsstaaten haben sich inzwischen alle 53 Staaten der Afrikanischen Union angeschlossen. ${ }^{3}$ Der Sitz des NePAD-Sekretariats mit rund 130 Mitarbeitern liegt in Pretoria, Südafrika. ${ }^{4}$

Bereits die Pläne Mbekis und Wades hatten für eine »Afrikanische Strategie für die Globalisierung « geworben, bei der die Hauptverantwortung für Afrikas Zukunft in erster Linie von den Afrikanern übernommen wird. Analog erkennt NePAD explizit an, dass es zunächst einmal Aufgabe der afrikanischen Staaten selbst und nicht der internationalen Politik - sei, sich den gemeinsamen Herausforderungen zu stellen. ${ }^{5}$ In diesem Sinne fokussiert NePAD auf eine Reihe von Prinzipien, $\mathrm{zu}$ deren wichtigsten gute Regierungsführung (Good Governance), afrikanische ownership, Partizipation der Bevölkerung sowie regionale und kontinentale Integration gehören und die durch einen differenzierten Entwicklungsplan erreicht werden sollen. Dieser Entwicklungsplan zielt insbesondere auf eine Verbesserung der wirtschaftspolitischen Rahmenbedingungen, auf die Sicherung makro-ökonomischer Stabilität sowie auf ein umfangreiches Investitionsprogramm zur Schließung bestehender Infrastrukturlücken.

(2) NePAD stellt nicht das erste ambitionierte gemeinsame Entwicklungsprogramm afrikanischer Staaten dar. Schon der Lagos Plan of Action von 1977, der Abuja-Vertrag von 1991 sowie weitere Beschlüsse und Initiativen der Organisation für Afrikanische Einheit (OAU) erklärten es zum Ziel, die wirtschaftliche und politische Kooperation, Entwicklung und Integration Afrikas entscheidend voranzutreiben. Die tatsächlichen Erfolge dieser Programme fielen jedoch ernüchternd aus. Das Scheitern dieser früheren Entwicklungsprogramme ist auch im Zusammenhang mit einigen für die afrikanische Politik bisher charakteristischen Merkmalen zu sehen. So herrschte als Ausdruck »pan-afrikanischer Elitensolidarität $\aleph^{6}$ zwischen den afrikanischen Staaten traditionell das »sakrosankte Prinzip der Nichteinmischung « ${ }^{7}$ und relativierte somit alle früheren Bekenntnisse zu länderübergreifender Kooperation und Good Governance in Afrika. ${ }^{8}$

3 Vgl. Cord Jakobeit, »Fünf Jahre NEPAD «, in: Aus Politik und Zeitgeschehen, 32-33/ 2006, S. 21-25; hier S. 21.

4 Vgl. http://www.nepad.org.

5 Dieses Bekenntnis zu afrikanischer Eigenverantwortung wird als zentrales Element der NePAD-Initiative gesehen. So z.B. Cord Jakobeit, FN 3, S. 21.

6 Ian Taylor, Can NEPAD Succeed Without Prior Political Reform? (Danish Institute for International Studies Working Paper 23), Copenhagen 2005; S. 15.

7 So die damalige G8-Beauftragte der Bundesregierung Uschi Eid, zitiert nach Helmut Matthes, »NEPAD - Zu den Veränderungen der Beziehungen zwischen den G8-Staaten und Afrika", in: Berichte (Januar 2005) des Forschungsinstituts der Internationalen Wissenschaftlichen Vereinigung Weltwirtschaft und Weltpolitik (IWVWW) e.V., Berlin 2005; S. 59.

8 Vgl. Henning Melber, »Glaubhaft geteilte Verantwortung?«, in: Entwicklung und Zusammenarbeit e+z, 4/2006, S. 160-162, hier S. 160. 
Vor diesem Hintergrund liegt die entscheidende Neuerung der NePAD-Initiative und der neu gegründeten Afrikanischen Union daher nicht in den angestrebten Zielen wie Wirtschaftswachstum und Stabilität, sondern vielmehr in den Mitteln und Verfahren, mit denen NePAD diese Ziele erreichen möchte. Eine wesentliche Neuerung der NePAD und der neu gegründeten afrikanischen Union liegt in diesem Sinne in einer Abkehr von den vormals eisernen Prinzipien der Nichtintervention und Nichteinmischung - zwei Prinzipien, die in der OAU noch als offizielle Norm galten. ${ }^{9}$ Im Rahmen der NePAD wird die Abkehr vom Prinzip der Nichteinmischung besonders deutlich am Instrument des African Peer Review Mechanism (APRM). Dieses Informations- und Kontrollsystem beruht auf einem gegenseitigen Beurteilungsprozess, anhand dessen überprüft werden soll, inwieweit die jeweiligen Regierungen die im Rahmen der NePAD festgelegten Ziele auch tatsächlich erreichen. Die Teilnahme am APRM ist freiwillig. Ende 2007 beteiligten sich 27 der insgesamt 53 NePAD-Mitgliedstaaten. ${ }^{10}$ Ein vom NePAD-Sekretariat unabhängiges APRMSekretariat ist für die Durchführung des APR-Prozesses verantwortlich. ${ }^{11}$

(3) Die Durchführung des African Peer Review Mechanism ist als mehrstufiges Verfahren angedacht. ${ }^{12}$ Eine besondere Rolle kommt hier dem Panel of Eminent Persons (APR Panel) zu; einer Gruppe »herausragender Persönlichkeiten « aus verschiedenen Ländern Afrikas. Ein Land beginnt seine Teilnahme am APRM-Prozess zunächst durch die Unterzeichnung eines Memorandum of Understanding mit dem APRM-Sekretariat in Pretoria. Dieses entsendet sodann ein Mitglied des APR Panel, das vor Ort bewertet, in welcher Form Vertreter aus Regierung, Zivilgesellschaft und Privatwirtschaft für das weitere Evaluierungsverfahren eingebunden werden sollen. In einem nächsten Schritt erstellen diese Vertreter eine Selbstevaluierung des betreffenden Landes (»self assessment «). Unter Leitung eines Mitglieds des Panel of Eminent Persons erarbeitet anschließend ein externes Überprüfungsteam (APR Team) einen eigenen Bericht. In einem letzten Schritt bespricht das externe Überprüfungsteam die Ergebnisse beider Vorberichte mit Regierungsvertretern des Landes und verfasst einen endgültigen Abschlussbericht. Dieser formuliert mit Blick auf die ermittelten Untersuchungsergebnisse Handlungsempfehlungen für die

9 Die Abkehr vom Prinzip der Nichtintervention kennzeichnet die neue Friedens- und Sicherheitsarchitektur der Afrikanischen Union, die im Fall akuter Krisensituationen erstmals die Möglichkeit kollektiver Interventionen in Mitgliedstaaten vorsieht. Im Fall der schon erfolgten Interventionen in Togo, der AU-Friedensmission in Darfur sowie des ausgehandelten Rückzugs von Charles Taylor wurde von dieser Möglichkeit auch bereits Gebrauch gemacht. Vgl. Konrad Melchers, »Editorial«, in: eins. Entwicklungspolitik Information Nord-Süd, 1-2/2006, S. 3 sowie Uschi Eid, »Afrikas Reformbemühungen ernst nehmen «, in: eins. Entwicklungspolitik Information Nord-Süd, 1-2/2006, S. 26-29, hier S. 29.

10 So die Angaben von NePAD im November 2007 unter http://www.nepad.org/aprm/.

11 Vgl. Ross Herbert, »The strengths and weaknesses of NePAD «, in: The Africa Report, 5/2007, S. 68-70, hier S. 69.

12 Vgl. http://www.nepad.org/aprm/. Für eine anschauliche Beschreibung des konkreten Verfahrens im Falle des APRM-Prozesses in Kenia vgl. Anyang Nyong'o, "Lohnenswert - Der Prozess des kenianischen >Peer Review`, in: eins. Entwicklungspolitik Information Nord-Süd, 1-2/2006, S. 30-31. 
jeweiligen Regierungen und berücksichtigt abschließend, inwiefern das NePADLand diesen Empfehlungen nachzukommen gedenkt. ${ }^{13}$

Im Vergleich zu früheren afrikanischen Entwicklungsinitiativen verspricht der African Peer Review Mechanism im Rahmen der NePAD einen qualitativ neuartigen Schritt. Wie das NePAD-Sekrariat selbst betont, bietet der APRM einen entscheidenden Ansatz, um wirtschaftliche Entwicklung, gute Regierungsführung und Demokratie zu stärken. Hierbei wird vor allem auch der Einbindung der Zivilgesellschaft große Bedeutung eingeräumt. Aus diesen Gründen hat der APRM hohe Erwartungen geweckt. ${ }^{14}$ Gleichzeitig finden sich jedoch auch zahlreiche Kritiker, die diese Erwartungen in Frage stellen. ${ }^{15}$ Einigkeit herrscht, dass der African Peer Review Mechanism wohl das innovativste und interessanteste Element der NePAD darstellt. ${ }^{16}$ Seine tatsächliche Leistungsfähigkeit bleibt jedoch umstritten.

Der folgende Abschnitt nutzt die Perspektive der Wirtschaftsethik sozialer Dilemmata, um genauer zu bestimmen, welches systematische Potential ein Selbstbindungsmechanismus wie der African Peer Revierw entfalten könnte. Ausgehend von diesen Überlegungen, erörtert der dritte Abschnitt, inwieweit die Erwartungen an den APRM auch tatsächlich erfüllt werden (können).

\section{Der»African Peer Review Mechanism « als Instrument der Selbstbindung}

Um das Potential des APRM näher zu bestimmen, gilt es, die zugrunde liegende Logik dieses Selbstbindungsmechanismus genauer zu entschlüsseln. Hierzu geht der folgende Abschnitt in zwei Schritten vor. Der erste Schritt nimmt das für afrikanische Staaten charakteristische System neo-patrimonialer Herrschaft in den Blick. Dieses Politiksystem, so die These, lässt sich als Unterentwicklungsfalle rekonstruieren: Das Ausbeutungsspiel zwischen Herrschenden und Bevölkerung führt in ein soziales Dilemma kollektiver Selbstschädigung. Der zweite Schritt zeigt auf, dass die Überwindung dieses Dilemmas eine Selbstbindung von Seiten der Herrschaftseliten voraussetzt. Angesichts der Strukturen schwacher Staatlichkeit sind die Möglichkeiten einer glaubwürdigen Bindung innerhalb der einzelnen Staaten jedoch be-

13 Dieses "Programme of Action « beschreibt den Aktionsplan, den das betroffene Land mit Blick auf die identifizierten Probleme in Angriff nehmen will. Ursprünglich war vorgesehen, dass dieser Plan alle aufgeworfenen Themen behandelt. Auf Druck Südafrikas wurde jedoch durchgesetzt, dass der Aktionsplan auch nur ausgewählte Probleme adressieren kann, die dann gemeinsam unter Einbindung der Zivilgesellschaft bearbeitet werden sollen. Vgl. Ross Herbert, FN 11, S. 70.

14 Einige Kommentatoren sehen in diesem Ansatz sogar »die bedeutendste Initiative, die jemals vorgebracht wurde, um den afrikanischen Kontinent von der Krise zur Erneuerung zu bewegen«; so der von Ian Taylor (FN 6, S.10) zitierte politische Beobachter Kempe R. Hope.

15 Vgl. statt vieler Ian Taylor (FN 6) sowie Patrick Chabal, »The quest for good government and development in Africa: is NEPAD the answer? «, in: International Affairs, (3) 2002, S. 447-462.

16 Vgl. Alex de Waal, FN 2, S. 471 sowie Cord Jakobeit, FN 3, S. 22. 
grenzt. Hier lässt sich der APRM als der Versuch interpretieren, Glaubwürdigkeit gleichsam von außen zu importieren.

(1) Das für viele afrikanische Staaten charakteristische Politiksystem kann als die Ausübung neo-patrimonialer Herrschaftsformen beschrieben werden ${ }^{17}$ : Unabhängig von den formalen politischen Strukturen wird hier Herrschaft über informale, klientelistische Beziehungen organisiert. Der herrschende Patron - sei es in der Person eines Autokraten, als Mitglied eines Clans oder allgemein als Stellvertreter einer Elite - erwirbt sich in diesem System Gefolgschaft, indem er Ressourcen an seine Anhänger umverteilt. Nicht die Orientierung am Gemeinwohl aller, sondern die Privilegierung der eigenen Klientel steht somit im Vordergrund der agierenden Patronagenetzwerke. ${ }^{18}$

Dieses neo-patrimoniale System steht nicht nur in Konflikt zu (westlichen) Vorstellungen von Demokratie und guter Regierungsführung. Es wird zudem als eine entscheidende Ursache für die fehlende wirtschaftliche Entwicklung in Afrika gesehen. ${ }^{19}$ Die drohende Aussicht, dass die Früchte der eigenen Arbeit durch die herrschende Elite umverteilt werden (könnten), untergräbt Erwartungssicherheit und führt dazu, dass die breite Bevölkerung in ein verändertes Konsum- und Investitionsverhalten ausweicht. ${ }^{20}$ Umverteilung bezeichnet dabei nicht nur offizielle staatliche Enteignungen, sondern erfolgt auch durch die willkürliche Erhebung von Gebühren und Steuern oder durch die Einforderung von Korruptionszahlungen. Die Gefahr, durch diese Mechanismen in der Zukunft ausgebeutet zu werden, führt in der Gegenwart zu starken Anreizen, zu konsumieren anstatt zu investieren. Investitionen bilden jedoch die entscheidende Voraussetzung für wirtschaftliches Wachstum. Besonders schwerwiegend ist, dass vor allem jene Investitionen unterlassen werden, die besonders ausbeutbar sind - etwa Investitionen in Bildung, in leistungsfähigere Maschinen, in die Verbesserung der landwirtschaftlichen Nutzfläche oder in arbeitsteilige Formen der Produktion. Dies hat gravierende Folgen, denn gerade die hier unterlassenen Investitionen sind für wirtschaftliche und gesellschaftliche Entwicklung von zentraler Bedeutung. ${ }^{21}$

In einer schematischen Vereinfachung lässt sich dieses System neo-patrimonialer Herrschaft als ein Ausbeutungsspiel zwischen zwei Gruppen rekonstruieren. ${ }^{22} \mathrm{Ab}-$

17 Vgl. Patrick Chabal, FN 15, S. 450 sowie Ian Taylor, Nepad. Toward Africa's Development or Another False Start, London, 2005, S. 2-3.

18 Vgl. Patrick Chabal, FN 15, S. 460.

19 Vgl. Patrick Chabal, FN 15, S. 453.

20 Für eine anschauliche und umfassende Darstellung der Anreize von Machthaber und Bevölkerung vgl. Mancur Olson, Power and Prosperity. Outgrowing Communist and Capitalist Dictatorships, New York 2000.

21 Im Gegensatz zu modernen, arbeitsteiligen Formen der Produktion erweist sich die (landwirtschaftliche) Subsistenzwirtschaft als vergleichsweise ausbeutungsresistent und nimmt in diesen Gesellschaften entsprechend eine zentrale Rolle ein.

22 Dieses Argument findet sich bereits bei Ingo Pies / Cora Voigt, »Demokratie in Afrika. Eine wirtschaftsethische Stellungnahme zur Initiative »New Partnership for Africa" (NePAD)«, in: Udo Ebert (Hrsg.): Wirtschaftsethische Perspektiven VIII. Grundsatzfragen, Unternebmensethik, Institutionen, Probleme internationaler Kooperation und nachhaltiger Entwicklung, Berlin 2006, S. 153-172. Der vorliegende Beitrag wählt eine alternative Darstellungsform, um diese Überlegungen weiterzuentwickeln. 
bildung 1 stellt die Logik dieses Spiels in einer Vier-Quadranten-Matrix dar. Diese Darstellung erlaubt es, die sich wiederholende Logik einer dynamischen Entscheidungssituation als gleichgewichtiges statisches Spiel zu modellieren. Die Rolle des Zeilenspielers kommt hier der Bevölkerung, aber auch inländischen und ausländischen Unternehmen zu. ${ }^{23}$ Bevölkerung und Unternehmen stehen vor der Wahl, Investitionen in dem Land zu tätigen (investieren) oder zu unterlassen (nicht investieren). Die Rolle des Spaltenspielers nimmt hingegen die Regierung ein. Diese vertritt nicht die Interessen der Gesamtbevölkerung, sondern die eines herrschenden Clans, eines Stammes, des Autokraten oder allgemein einer Elite. Dieses Regime bekennt sich zwar offiziell zu den Prinzipien von Demokratie und Good Governance. De facto steht es jedoch vor der Wahl zwischen zwei Strategien. Die erste Strategie besteht darin, das eigene Bekenntnis zu Demokratie und Good Governance zu unterlaufen und stattdessen durch Korruption, Enteignung oder sonstigen Machtmissbrauch die Investitionserträge von Bevölkerung und Unternehmen an die eigene Klientel umzuverteilen (Machtmissbrauch). Die zweite Strategie besteht darin, die Kriterien guter Regierungsführung tatsächlich einzuhalten, die eigene Macht einzuschränken und auf die Ausbeutung der Bevölkerung zu verzichten (Good Governance).

Abbildung 1: Das Ausbeutungsspiel neo-patrimonialer Herrschaft als Rationalfalle

\section{Regierung}

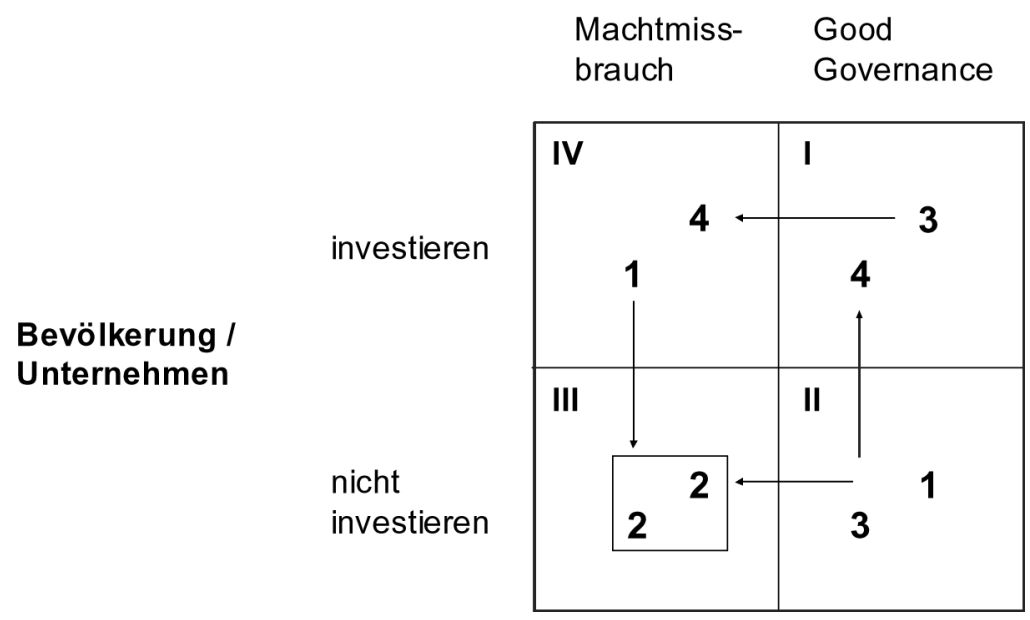

23 Nur mit Blick auf das betrachtete Problem ist es zulässig, diese äußerst heterogenen Akteure als eine homogene Gruppe zu modellieren: Drohender Machtmissbrauch beeinflusst den Wahlkalkül sämtlicher Akteure, die vor - ausbeutbaren - Investitionsentscheidungen stehen. 
Betrachtet man nun die vier denkbaren Strategiekombinationen, ergeben sich aus der Sicht beider Spieler folgende individuelle Auszahlungen.. ${ }^{24}$ Das subjektiv beste Ergebnis für die herrschende Gruppe (Payoff $=4)$ ergibt sich in Quadrant IV. Hier tätigen Bevölkerung und Unternehmen Investitionen, deren Erträge sich die Regierung durch Machtmissbrauch aneignen kann. Das umgekehrt schlechteste Ergebnis (Payoff $=1$ ) ergibt sich aus Sicht der Herrscherelite in Quadrant II. Hier verzichtet sie auf die Machtprivilegien und sieht sich gleichzeitig fehlenden Investitionen gegenüber. Wie sieht nun die Bewertung der Quadranten I und III aus Sicht der Herrscherelite aus? Hier kommt folgende Überlegung zum Tragen: Wenn die herrschende Elite nicht nur durch Umverteilungen, sondern auch durch die eigene Teilnahme am Markt ein Einkommen erzielt, hat sie ein Interesse an allgemeiner wirtschaftlicher Prosperität. ${ }^{25}$ Aus diesem Grund wird sie die Teilnahme am Wirtschaftswachstum in Quadrant I (Payoff $=3$ ) dem Quadranten III (Payoff $=2$ ) vorziehen, in dem sie ihre Ausbeutungsmacht zwar theoretisch bewahrt - faktisch jedoch gar keine ausbeutbaren Investitionen getätigt werden. Ein anderes Bild zeigt sich aus Sicht der Bevölkerung. Für sie besteht die beste Strategiekombination in Quadrant I (Payoff $=4$ ), in dem sie Investitionen tätigen kann, ohne dass diese ausgebeutet werden. Im Vergleich hierzu ist es für die Bevölkerung weniger attraktiv, an sich gewinnbringende Investitionen zu unterlassen (Quadrant II und III). In diesem Fall der Unterlassung von Investitionen würde sie die Einhaltung von Good Governance der Regierung in Quadrant II (Payoff $=3$ ) dem Machtmissbrauch in Quadranten III (Payoff $=2)$ vorziehen. Das schlechteste Ergebnis stellt sich aus Sicht der Bevölkerung jedoch in Quadrant IV ein (Payoff $=1$ ). Hier investieren Bevölkerung und Unternehmen eigene Ressourcen; die Erträge dieser Investitionen werden jedoch an die herrschende Elite umverteilt.

Beide Spieler, Bevölkerung und Herrscherelite, werden nun versuchen, der Strategiewahl des anderen bestmöglich zu begegnen. Die eingezeichneten Pfeile in Abbildung 1 spiegeln diese individuellen Vorteils-Nachteils-Überlegungen wider. Die vertikalen Pfeile illustrieren den Rationalkalkül der Bevölkerung. Ihr Interesse besteht darin, nur dann zu investieren, wenn die Regierung die Prinzipien von Good Governance tatsächlich einhält. Folglich weist der linke Pfeil nach unten und der rechte Pfeil nach oben. Die horizontalen Pfeile verdeutlichen hingegen den Rationalkalkül der Regierung. Sie illustrieren, dass die Regierung unabhängig vom Verhalten der Bevölkerung stets ein Interesse daran hat, ihre Macht nicht einzuschränken. Beide horizontalen Pfeile weisen daher nach links.

Zusammen genommen führen diese Rationalkalküle zu folgendem Ergebnis. Quadrant III ist der einzige Quadrant, in den zwei Pfeile hineinweisen. Spieltheoretisch formuliert, liegt hier ein Nash-Gleichgewicht vor: Ist diese Strategiekombina-

24 Die in den Quadranten jeweils vordere Zahl steht für den ordinalen Payoff von Bevölkerung und Unternehmen. Die Zahl hinter dem Komma repräsentiert die Auszahlung von Autokrat bzw. Herrschaftselite. Die subjektiv beste Strategiekombination wird mit 4 bewertet, die zweit- und drittbeste mit 3 bzw. 2. Die viertbeste und zugleich schlechteste Strategiekombination erhält den Wert 1. Es gilt: $4>3>2>1$.

25 Vgl. Mancur Olson, FN 22, S. 16-17. 
tion einmal erreicht, gibt es keine individuellen Anreize mehr, von ihr abzuweichen. Weder für die Bevölkerung noch für die Regierung ist es rational, eine andere Strategie zu wählen. ${ }^{26}$ Obwohl sich beide Spieler individuell rational verhalten, realisieren sie ein stabiles Gruppenergebnis, mit dem sie sich selbst schädigen: Wie ein Vergleich der Pay-offs zeigt, könnten sich beide Spieler besser stellen, wenn sie gemeinsam den Quadranten I statt den Quadranten III erreichten. Rekonstruiert als Ausbeutungsspiel, weist das System neo-patrimonialer Herrschaft somit die Struktur eines Quasi-PD²7 auf, also eines sozialen Dilemmas: Individuell rationales Verhalten führt hier zu einer kollektiven Selbstschädigung.

Die Logik des Systems neo-patrimonialer Herrschaft beschreibt folglich eine Rationalfalle: Für die herrschende Elite erscheint es rational, ihre Macht nicht einzuschränken. Für die Bevölkerung ist es rational, keine ausbeutbaren Investitionen zu tätigen. Gleichzeitig schädigt die resultierende wirtschaftliche Unterentwicklung alle Beteiligten. ${ }^{28}$ Das Phänomen fehlender Wirtschaftsentwicklung erweist sich somit zwar als pareto-inferior, aber auch als langfristig stabil. Vor diesem Hintergrund gilt es nun zu prüfen, inwieweit der African Peer Review Mechanism als Versuch politischer Entrepreneurs interpretiert werden kann, die Logik dieses sozialen Dilemmas zu durchbrechen.

26 Mit Blick auf diesen Rationalkalkül der Regierung formuliert Ian Taylor (FN 6, S. 2) eine Kritik an den offiziellen NePAD-Bekenntnissen wie etwa zu Good Governance: »The irony is that the type of solutions [good governance, M.B.] advanced by Nepad would deprive rulers of the means to maintain their patronage networks. [...] Nepad seems to advance the idea that the very same African elites who benefit from the neopatrimonial state will now commit a form of class suicide «. Taylor verweist somit darauf, dass es aus Sicht der neo-patrimonialen Elite überhaupt nicht rational wäre, das Bekenntnisse zu Good Governance auch tatsächlich einzuhalten. Dieser Einschätzung ist innerbalb des gegebenen Spiels zuzustimmen. Im Folgenden wird jedoch gezeigt, dass das eigentliche Potential des APRM darin liegt, ein grundsätzlich transformiertes, besseres Spiel zu spielen.

27 Vgl. hierzu grundlegend Ingo Pies, Ordnungspolitik in der Demokratie, Tübingen 2000, S. 223 f. PD steht hier für das zweiseitige Gefangenendilemma (»prisoners' dilemma«). Die Gemeinsamkeit zwischen einem echten PD und einem Quasi-PD liegt darin, dass beide Fälle ein soziales Dilemma beschreiben: Die Nash-Gleichgewichtslösung ist pareto-inferior. Der Unterschied zwischen PD und Quasi-PD besteht in der Asymmetrie des Quasi-PD: Während im PD beide Spieler eine dominante Strategie verfolgen, gilt dies im hier beschriebenen Fall des Neo-Patrimonialismus nur für die Regierungsseite. Für diese ist es stets die dominante Strategie, das Bekenntnis zu Good Governance zu unterlaufen. Dieser Unterschied ist wichtig, weil die Überwindung des sozialen Dilemmas im Quasi-PD eine andere Lösung erfordert als im zweiseitigen PD. Während im PD die Anreizstruktur für beide Seiten geändert werden muss, reicht im Quasi-PD die Änderung der Anreize einer Seite, um den für beide Seiten vorteilhaften Quadranten in ein Nash-Gleichgewicht zu überführen.

28 Implizit findet sich diese Einschätzung auch bei Patrick Chabal (FN 15, S 454, eigene Hervorhebung). Dieser beurteilt die Logik des Systems neo-patrimonialer Herrschaft wie folgt: »Thus, the primacy of communitarian and clientelistic political imperatives which may make good sense at the micro-level of individuals and communities - leads assuredly to massive economic inefficiency and, possibly, to terminal damage at the national level.« 
(2) Nicht nur die Bevölkerung, sondern auch die regierende Elite stellt sich in der Unterentwicklungsfalle des neo-patrimonialen Systems langfristig schlechter. Aus diesem Grund läge es im Interesse der herrschenden Elite, auf die eigene Ausbeutungsoption zu verzichten, die allgemeine Erwartungssicherheit zu erhöhen und so das wechselseitig vorteilhafte Wirtschaftswachstum in Quadrant I zu realisieren. Hier zeigt sich jedoch ein entscheidendes Umsetzungsproblem. Die bloße Ankündigung, die Prinzipien guter Regierungsführung einzuhalten, ist schlichtweg nicht glaubwürdig. Zugrunde liegt ein Zeitinkonsistenzproblem. ${ }^{29}$ Zwar hat die Regierung bzw. die dahinter stehende Elite $e x$ ante ein Interesse daran, auf ihren wachstumsverhindernden Machteinfluss zu verzichten. Sobald es jedoch tatsächlich zu einem durch diese Ankündigung bewirkten Wachstum kommt, besteht ihr Interesse ex post unvermindert darin, sich diese Investitionserträge durch Machtmissbrauch anzueignen. ${ }^{30}$

Dieses Zeitinkonsistenzproblem wird zusätzlich durch die besondere Interaktionsdynamik auf Seiten der Herrschenden verschärft. In früheren ökonomischen Modellen wurde hier oftmals ein Autokrat angenommen, der als Einzelperson versucht, seinen Nutzen zu maximieren. Im System neo-patrimonialer Herrschaft hängt die Macht des Patrons jedoch entscheidend davon ab, die Gefolgschaft der Elite durch Umverteilung von Ressourcen an sie zu sichern. ${ }^{31}$ In diesem Sinn kann der Autokrat oder eine Regierung auch als Agent betrachtet werden, der im Auftrag eines Prinzipals - hier: der privilegierten Elite - handelt. ${ }^{32}$ Ein Autokrat steht daher unter starkem Druck, konsequent Ressourcen an seine Anhänger umzuverteilen. Andernfalls droht ihm die Gefahr, dass ihn die herrschende Elite selbst - auch mit Gewalt - durch einen anderen Autokraten ersetzt, der der Elite eine lukrativere Umverteilung verspricht. Die hieraus resultierende Kurzfristorientierung schränkt den Handlungsspielraum des Autokraten ein und führt zusätzlich dazu, dass sein Bekenntnis zu Good Governance und zu einer langfristigen Wachstumsorientierung wenig glaubhaft ist. ${ }^{33}$

29 Die durch das Phänomen der Zeitinkonsistenz auftretenden Bindungsprobleme beschreibt bereits ausführlich Thomas Schelling, The Strategy of Conflict, Cambridge 1960, insb. Kapitel 2 sowie anschaulich auch Thomas Schelling, Strategies of Commitment and Other Essays, Cambridge 2006.

30 Vgl. Ingo Pies / Cora Voigt, FN 22, S. 162.

31 Vgl. Patrick Chabal, FN 15, S. 453.

32 Vgl. Ingo Pies / Cora Voigt, FN 22, S. 158.

33 Diesen Zwang der Kurzfristorientierung beschreibt auch Patrick Chabal, FN 15, S. 453. Er fasst zusammen: »Indeed, neo-patrimonialism may well have reached its limits; or rather, if may now have been changed into a political economy of sdisorder . Where the search for short-term economic gain is paramount, political leaders find that their legitimacy as Big Men is conditional upon their ability to obtain resources - by all means.« 
Abbildung 2: Überwindung des Quasi-PD durch individuelle Selbstbindung

\section{Regierung}

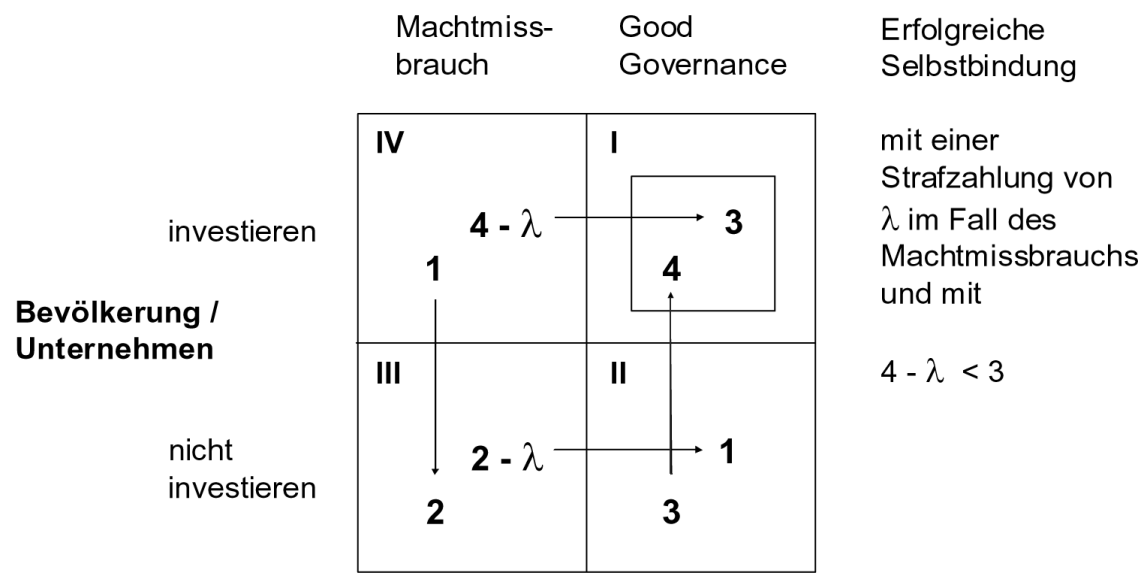

Das Zeitinkonsistenzproblem lässt sich nur überwinden, wenn die Regierung ihre eigene Ankündigung durch eine sanktionsbewehrte Selbstbindung glaubhaft macht. ${ }^{34}$ Eine solche Selbstbindung wird dann glaubhaft, wenn durch sie der Bruch des eigenen Versprechens für die Regierung teuer wird. Sind die Folgen eines gebrochenen Versprechens hinreichend unangenehm, dann ist es für die Regierung nicht mehr attraktiv, ihre Macht - auf teure Weise! - zu missbrauchen. Abbildung 2 illustriert dies erneut anhand einer Vier-Quadranten-Matrix. In dieser Darstellung ist der Bruch des eigenen Versprechens für die herrschende Regierung aufgrund eines Sanktionsmechanismus' mit den Kosten $\lambda$ verbunden. Ist $\lambda$ hinreichend groß (hier: $\lambda>1$ ), dann verliert die Ausbeutungsoption für die Regierung so sehr an Attraktivität, dass es für sie rational wird, die Prinzipien von Good Governance einzuhalten. ${ }^{35}$ Das neue Nash-Gleichgewicht liegt nun im Quadranten I. Eine wechselseitige Besserstellung für die herrschende Elite und die Bevölkerung wird möglich.

So einfach die Lösung der Unterentwicklungsfalle in der (Spiel-)Theorie modelliert werden kann, so schwierig gestaltet sich eine Lösung unter den empirischen Bedingungen vieler afrikanischer Staaten. Denn eine politische Selbstbindung der

34 Zur Konzeption von Selbstbindungen vgl. grundlegend Jon Elster, Ulysses and the sirens. Studies in rationality and irrationality, Cambridge 1979; Jon Elster, Subversion der Rationalität, Frankfurt 1987. Die Thematik der Selbstbindung behandelt auch Klaus Dieter Wolf, etwa in Klaus Dieter Wolf, »Entdemokratisierung durch Selbstbindung in der Europäischen Union«, in: Klaus Dieter Wolf (Hrsg.): Projekt Europa im Übergang? Probleme, Modelle und Strategien des Regierens in der Europäischen Union, Baden-Baden 1997, S. 271-294.

35 Graphisch ausgedrückt, zeigen die Pfeile, die die Vorteils-Nachteils-Überlegungen der Regierung illustrieren, nun von links nach rechts. 
herrschenden Elite setzt glaubhafte und sanktionsbewehrte Bindungsmechanismen voraus. Diese Bindungstechnologien sind jedoch nicht universal gegeben. Vielmehr hängt ihre Verfügbarkeit ab von einer Vielzahl höchst kontingenter gesellschaftlicher Institutionen. ${ }^{36}$ Zwei Punkte mögen illustrieren, dass viele Problemlösungsverfahren, die beispielsweise in westlichen Demokratien gut funktionieren, oft nur begrenzt unter den politischen Bedingungen in Afrika anwendbar sind. Erstens kann die Selbstbindung einer Regierung glaubhaft sein, wenn sie im Falle eines gebrochenen Versprechens mit massiven Protesten aus der Zivilgesellschaft rechnen muss. Dies setzt unabhängige Medien, gut organisierte Bürgerinteressen und weitgehende politische Freiheiten voraus - Bedingungen, die in vielen Entwicklungsländern oft gerade nicht gegeben sind. ${ }^{37}$ Auch die Sanktionsfunktion von Wahlen ist eingeschränkt, wenn diese nicht frei und unabhängig durchgeführt werden. Zweitens können Regierungen in einer westlichen Demokratie auf den Verfassungsstaat und eine funktionierende Gewaltenteilung zurückgreifen. Selbstbindungen der Regierung sind hier in Form von Recht oder Verfassungsänderungen möglich, die durch Instanzen wie etwa die Verfassungsgerichte auch sanktionsbewehrt durchgesetzt werden. Im System neo-patrimonialer Herrschaft wird die Funktionsfähigkeit der formalen politischen Institutionen, die zuallererst Rechtsstaatlichkeit, Gewaltenteilung und Verfassungsgerichtsbarkeit ermöglich sollen, jedoch de facto untergraben. ${ }^{38}$ Selbstbindungen durch Recht sind hier nur bedingt glaubhaft. Die scheinbar unbegrenzte Macht der herrschenden Elite erweist sich hier letztlich als Ohnmacht.

Vor diesem Hintergrund kann nun abschließend das besondere Potential des African Peer Review Mechanism gewürdigt werden. Zugespitzt formuliert, erwächst die potentielle Stärke dieses länderübergreifenden Informations- und Kontrollsystems aus der ausgeprägten Schwäche der internen Bindungsfähigkeit afrikanischer Staaten. Nach Jahrzehnten von Korruption und autokratischem Machtmissbrauch stehen selbst progressive Regierungen vor dem Problem, ihre Reformanstrengungen glaubhaft zu machen. Angesichts fehlender geeigneter Bindungsmöglichkeiten innerhalb ihrer Staaten lässt sich der African Peer Review Mechanism als der Versuch politischer Entrepreneurs werten, aus dem eigenen Land herauszutreten und Glaubwürdigkeit gleichsam von außen zu importieren. Hier geht es darum, eine hinreichend teure Sanktion $\lambda$ für den Fall zu schaffen, dass eine Regierung ihre

36 Vgl. etwa Markus Beckmann / Ingo Pies, Responsibility and Economics (Diskussionspapier Nr. 2007-6 des Lehrstuhls für Wirtschaftsethik an der Martin-Luther-Universität Halle-Wittenberg, hrsg. von Ingo Pies), Halle 2007, insb. S. 20-23.

37 Trotz einer in den letzten Jahren prosperierenden NGO-Szene in Afrika sind die strukturellen Unterschiede zur Zivilgesellschaft in westlichen Nationalstaaten zu beachten. So schreibt Patrick Chabal, FN 15, S. 461: »The currently fashionable view that the impetus for democratic change will come from >civil society< is, therefore, based on wishful thinking [for at least two reasons:] The first is that the development of a strong, autonomous and politically articulate civil society is the consequence of social differentiation based on economic development. The second is that civil society can act to foil the state only where the state is institutionally differentiated from society. Neither of these conditions applies to contemporary Africa."

38 Vgl. Patrick Chabal, FN 15, S. 460. 
Selbstverpflichtung zu Good Governance brechen würde, um sich die Investitionserträge von Unternehmen und Bevölkerung anzueignen. Der kollektive African Peer Review-Prozess kann somit als ein Meta-Spiel betrachtet werden, das die Anreize in den einzelnen »Länderspielen « so verändern soll, dass eine wechselseitige Besserstellung von Bevölkerung und Eliten möglich wird. Diesem Meta-Spiel gleichsam »oberhalb« des einzelnen Staates kommt gerade deshalb eine besondere Bedeutung zu, weil die Möglichkeiten erfolgreicher Meta-Spiele innerhalb der jeweiligen Staaten begrenzt sind. Mit Blick auf dieses systematische Potential gilt es im folgenden Abschnitt zu diskutieren, inwiefern diese Erwartungen an den APRM in der Umsetzung erfüllt werden (können).

\section{Stärken und Schwächen des APRM}

Rekonstruiert aus Sicht der Wirtschaftsethik sozialer Dilemmata, resultiert das systematische Potential des African Peer Review Mechanim aus einer Selbstbindungslogik: In der Interaktion mit anderen kann sich die eigene Bindung, richtig verstanden, als produktiv erweisen. Diese Konzeptualisierung erlaubt es, zu den Stärken und Schwächen des APRM in seiner tatsächlichen Umsetzung Stellung zu nehmen. Hierzu greift dieser Abschnitt eine Reihe aktuell diskutierter Fragen auf und erörtert die Bedeutung der Integrität des APRM-Verfahrens (1), die Einbindung der Zivilgesellschaft (2), die Rolle externer (wirtschaftlicher) Unterstützung (3) sowie die Frage nach der Inklusivität des APRM-Prozesses ((4) und (5)).

(1) Die Selbstbindungslogik des APRM funktioniert nur dann, wenn das Brechen des eigenen Versprechens hinreichend unattraktiv ist. Aus diesem Grund bemängeln Kritiker, dass der APRM im Falle einer Nichtbeachtung der Prinzipien guter Regierungsführung keinerlei harte Sanktionen vorsieht. ${ }^{39}$ Anders formuliert, erscheint aus dieser Sicht $\lambda$ so klein, dass kein Wechsel des Verhaltensgleichgewichts möglich wird. NePAD selbst hebt hervor, dass der Peer Review nicht als Bestrafungsinstrument, sondern als Lern- und Korrekturmechanismus gedacht ist. ${ }^{40}$ Die mögliche Bindungswirkung des APRM beruht daher primär auf einem Reputationsmechanismus, der über naming und shaming weichen Druck erzeugt. ${ }^{41}$ Aus diesem Grund kommt der Integrität und Transparenz des APRM-Prozesses eine zentrale Bedeutung zu. Je größer die Integrität des Prozesses, desto größer ist die Bindungswirkung des APRM.

Hier zeigt sich eine Reihe von Schwächen in der bisherigen Umsetzung des APRM. Erstens verweisen Kritiker darauf, dass bereits die personelle Besetzung wichtiger Positionen im NePAD- und APRM-Prozess die Glaubwürdigkeit des Verfahrens untergrabe. So umfasse das NePAD Heads of State Implementing Committee (HSIC), das unter anderem den endgültigen APRM-Länderbericht billigt,

39 Vgl. Ian Taylor, FN 6, S. 12.

40 So erklärt der NePAD-Internet-Auftritt: »The APRM is not meant to exclude or punish countries. There is no conditionality attached to the mechanism". http:// www.nepad.org/aprm/.

41 Vgl. Cord Jakobeit, FN 3, S. 22. 
mit Paul Biya (Kamerun), Denis Sassou-Nguesso (Kongo) und Omar Bongo (Gabun) eine Reihe von Staatsführern, die ihrerseits die Grundsätze von Good Governance und Demokratie massiv missachten. ${ }^{42}$ In gleicher Weise wird die Unabhängigkeit der Mitglieder des Eminent Persons Panel in Frage gestellt. ${ }^{43}$ Kurz, es bestehen Zweifel an der persönlichen Integrität der am APRM Beteiligten.

Als zweite Schwäche werden strukturelle Defizite des APR-Verfahrens genannt. In der Anwendung erweist sich dieser Prozess als vergleichsweise kompliziert und zeitaufwendig. Gleichzeitig fehlen dem APRM-Sekretariat ausreichende Kapazitäten, um die Monitoring-Prozesse innerhalb der vorgesehenen Zeitpläne tatsächlich umzusetzen. Zusätzlich bestehen zum Teil widersprüchliche Regelungen. ${ }^{44}$

Eine dritte Schwäche wird darin gesehen, dass die betroffenen Regierungen großen Einfluss auf den APRM-Prozess in ihrem Land ausüben können. In der Tat haben zahlreiche Regierungen das Mandat und den Anwendungsbereich des APRM auf ihr Land stark eingeschränkt. ${ }^{45}$ Durch die mangelnde Ausstattung des APRM mit finanziellen Ressourcen ergibt sich eine zusätzliche Abhängigkeit von der jeweils zu beurteilenden Regierung. ${ }^{46}$ Auch sieht der APRM-Fahrplan vor, dass die Regierungen bei der Erstellung des Abschlussberichts intensiv eingebunden werden. ${ }^{47}$ Die Unabhängigkeit dieses Dokuments erscheint somit zweifelhaft. Darüber hinaus steht es den Regierungen am Ende frei zu entscheiden, ob der Abschlussbericht überhaupt veröffentlicht werden darf. ${ }^{48}$ Unliebsame Ergebnisse können so unter Verschluss gehalten werden.

Trotz der berechtigten Kritik kann der vorhandene Freiheitsraum der zu beurteilenden Regierung jedoch nicht nur als Schwäche, sondern auch als eine mögliche Stärke des APRM gewertet werden. So haben sich mit Ghana, Ruanda und Mauritius die ersten Länder, die den APRM-Prozess durchlaufen, freiwillig darauf verpflichtet, nicht nur den von der Regierung möglicherweise geschönten Abschlussbericht, sondern auch die unter Einbindung der Zivilgesellschaft erstellte Selbstevaluierung des betreffenden Landes sowie den Bericht des externen Überprüfungsteams zu veröffentlichen. ${ }^{49}$ Aufgrund der Freiwilligkeit dieser Maßnahme

42 Vgl. Ian Taylor, FN 17, S. 155-156.

43 Ian Taylor (FN 6, S.16) urteilt etwa: »All of these panellists are intimately linked to the elite classes of Africa and [that] brings into question any notion of real independence and objectivity. ... Indeed, the whole Eminent Persons panel is devoid of credibility."

44 So sehen die schriftlichen APRM-Richtlinien beispielsweise vor, dass innerhalb eines Landes die betroffene Regierung den internen Evaluierungsprozess organisiert - ein Verfahren, wie es etwa in Ruanda und Südafrika verwendet wurde. Das Panel der »Eminent Persons « machte es dagegen zur Auflage, dass die nationalen Evaluierungskommissionen von Wirtschaft und Zivilgesellschaft geleitet wurden, um so eine größere Unabhängigkeit von der Regierung zu garantieren. Dieses Verfahren kam wiederum in Ghana und Kenia zur Anwendung. Vgl. Ross Herbert, FN 11, S. 70.

45 Vgl. Henning Melber, FN 8, S. 160.

46 Vgl. etwa Ian Taylor, FN 6, S. 12 sowie Ross Herbert, FN 11, S. 70.

47 Vgl. NEPAD Secretariat, The African Peer Review Mechanism (APRM) (2003), in: http://www.nepad.org; (Stand: 07.11.2007).

48 Vgl. Ian Taylor, FN 6, S. 11.

49 Vgl. Konrad Melchers, FN 9, S. 3. 
stellt diese Selbstverpflichtung ein zusätzliches Signal dar, den Reformprozess tatsächlich ernst zu nehmen. Dies kann helfen, die Bindungswirkung des APRM für diese Länder sogar zu erhöhen.

(2) Die Tatsache, dass NePAD zunächst in erster Linie den westlichen Geberländern, aber kaum der eigenen Bevölkerung präsentiert wurde, weckt $Z$ weifel an der Glaubwürdigkeit des NePAD-Projekts. ${ }^{50} \mathrm{NePAD}$, so der Vorwurf, stelle ein von der breiten Bevölkerung kaum wahrgenommenes Elitenprojekt dar. ${ }^{51}$ Für die verbreitete Forderung, die Zivilgesellschaft vor Ort aktiver in den NePAD- und insbesondere den APRM-Prozesss einzubinden, spricht folglich gleich eine Reihe von Gründen. Erstens stellt die Einbindung unterschiedlicher Stakeholder den APRMProzess auf eine breitere Informationsbasis und hilft so, die Qualität der Länderberichte zu verbessern. Zweitens erhöht die Einbeziehung nichtstaatlicher Akteure die Legitimität und Glaubwürdigkeit dieser Berichte. ${ }^{52}$ Zuguterletzt bringt gerade die gezielte Einbindung der Bevölkerung das eigentliche Potential der Selbstbindung zur Geltung: Die Partizipation der Zivilgesellschaft stellt das vielleicht wirksamste Instrument dar, die Ernsthaftigkeit der eigenen Reformbemühungen gerade gegenüber jener Zielgruppe zu dokumentieren, der Erwartungssicherheit signalisiert werden soll: der eigenen Bevölkerung. ${ }^{53}$ Wie weit Partizipation tatsächlich reicht, entscheiden im konkreten Fall vielmehr die betroffenen nationalen Regierungen. Die fehlenden Standards für die Einbindung der Zivilgesellschaft innerhalb des APRM stellen folglich eine entscheidende Schwäche dieses Verfahrens dar.

(3) Versteht man den APRM als afrikanischen Selbstbindungsmechanismus, lautet eine entscheidende Frage, ob - und wenn ja: wie - die Industriestaaten NePAD und den APRM-Prozess aktiv unterstützen sollen. Hier lassen sich drei Punkte festhalten.

Erstens können die Industriestaaten helfen, die Kapazitäten des APRM zu stärken und so die Effektivität dieses Bindungsmechanismus' zu erhöhen. Denkbar sind hier ein gezielter Wissenstransfer, logistische Unterstützung, die Bereitstellung finanzieller Ressourcen für das APRM-Sekretariat, die Schulung und Weiterbildung von APRM-Mitarbeitern und zivilgesellschaftlichen Vertretern sowie der Aufbau von Systemen zur Datenerfassung und Datenverarbeitung. ${ }^{54}$ Je verlässlicher der APRM als »Bindungstechnologie« ist, desto eher besteht für afrikanische Eliten Planungs- und Erwartungssicherheit, um dieses Instrument zu nutzen.

Zweitens gilt es, NePAD als einen dezidiert politischen (Lern-)Prozess zu unterstützen. Die Zusammenarbeit zwischen G8- und NePAD-Staaten entfaltet hier eine wichtige Ankerfunktion. Die Rückbindung an die internationale Gemeinschaft

50 So bemerkt Alex de Waal, FN 2, S. 463: »But few [of the NePAD leaders] haven taken the trouble to explain to their citizens the basic principles of NePAD, an omission that has led to much speculation about its purpose and content.«

51 Vgl. Cord Jakobeit, FN 3, S. 25.

52 Vgl. Henning Melber, FN 8, S. 161.

53 In diesem Sinne wäre analog zu überlegen, wie auch nationale wie multinationale Unternehmen (stärker) in den Prozess des APRM eingebunden werden können.

54 Vgl. Ingo Pies / Cora Voigt, FN 22, S. 167. 
kann die Einhaltung der angekündigten Selbstbindungen mit zusätzlichem weichem Druck flankieren. Diese politischen Gespräche sollten auf absehbare Zeit fortgesetzt werden. ${ }^{55}$

Der dritte und wohl umstrittenste Punkt betrifft die Vergabe wirtschaftlicher Hilfe. Hier gibt es unterschiedliche Ansichten. Die einen argumentieren, dass es eines big push bedarf, also eines Schubs deutlich aufgestockter Entwicklungshilfe, um die Reformbemühungen in Afrika wirksam zu unterstützen. Im Vordergrund steht hier die Armutsbekämpfung. ${ }^{56}$ Andere halten entgegen, dass die Fokussierung auf die Beseitigung wirtschaftlicher Defizite den NePAD-Prozess entpolitisiert und so dessen eigentliches Potential gefährdet. ${ }^{57}$ Einige Skeptiker bezweifeln von vorneherein dieses Potential und sehen in NePAD lediglich den (geschickten) Versuch, das alte Geber-Empfänger-Schema unter einer neuen Überschrift fortzusetzen. ${ }^{58} \mathrm{Zugu}$ terletzt gibt es auch eine moralische Kritik. Diese lehnt es ab, Staatsführer für die Einhaltung von Good-Governance-Prinzipien zu belohnen, zu denen die afrikanischen Regierungen ohnehin auch ohne die Unterstützung des Nordens verpflichtet seien..$^{59}$

Hier ist eine differenzierte Betrachtung nötig. Die bisher entwickelten Überlegungen verdeutlichen, dass für die Umsetzung von Good Governance nicht nur das Wollen der Regierung, sondern auch ihr Können von entscheidender Bedeutung ist. Die differenzierte Frage lautet daher: Wie kann wirtschaftliche Hilfe das Bindungsvermögen afrikanischer Regierungen - hier: die Selbstbindungswirkung des APRM - sinnvoll unterstützen? Aus dieser Sicht ist die Strategie eines big push kritisch zu betrachten. Die (unkonditionierte) Zuführung beträchtlicher Entwicklungsgelder schafft Anreize, sich diese zusätzlichen Ressourcen durch Machtmissbrauch anzueignen. Gleichzeitig mindert die Aussicht auf exogen generiertes Wachstum für die Regierung den Anreiz, sich selbst so zu binden, dass endogenes Wachstum möglich wird. Die Strategie eines big push birgt folglich die Gefahr, die bestehenden Strukturen neo-patrimonialer Herrschaft langfristig sogar zu stabilisieren. ${ }^{60}$

55 Vgl. Uschi Eid, FN 9, S. 28.

56 Im Bereich der Politik ließe sich dieser Ansatz in den letzten Jahren tendenziell etwa der britischen Regierung zuschreiben. Für einen prominenten Befürworter aus der Wissenschaft vgl. Jeffrey D. Sachs, The End of Poverty. Economic Possibilities of our Time, New York 2005.

57 Vgl. Uschi Eid, FN 9, S. 28.

58 So schreibt Patrick Chabal, FN 15, S. 462: »NEPAD must, in large measure at least, be understood as a commitment on the part of the current (and not so new) elites in Africa to the present >democratic orthodoxy< in order to guarantee a transfer of resources to Africa: a continuation with, rather than a break from, the type or relations that has guided the continent's engagement with the international community since independence."

59 Diese Position findet sich etwa bei Taylor (FN 6, S. 19), der kritisiert: »Rewarding< leaders with extra resources for not undermining human rights and not preventing democracy is flawed. ... African leaders should be encouraging the practice of good governance irrespective of the stance of the industrialized states «. Ähnlich auch Ian Taylor, FN 17, S. 158.

60 Vgl. Ingo Pies / Cora Voigt, FN 22, S. 163. 
Welche Möglichkeiten gibt es nun, die Selbstbindungswirkung des APRM durch wirtschaftliche Unterstützung zu flankieren? Hier kommen eine direkte und eine indirekte Strategie in Betracht. Die direkte Strategie besteht in der Zahlung von Entwicklungsgeldern, für deren Vergabe jedoch besondere Kriterien für best practices politischer Selbstbindung zugrunde gelegt werden. ${ }^{61} \mathrm{Z}$ war findet das Prinzip der Konditionierung in einigen Bereichen der Entwicklungszusammenarbeit seit Jahren Anwendung. Hier mehren sich jedoch Zweifel hinsichtlich der Effektivität und Legitimation dieser von außen vorgegebenen Konditionen. ${ }^{62}$ Der APRM kann einen Ansatz bieten, effektivere Kriterien für die Vergabe zusätzlicher Entwicklungsgelder aus den betroffenen Staaten selbst zu entwickeln..$^{63}$

Die indirekte, langfristig wohl aber wirksamere Strategie besteht darin, die Bedingungen für eigenes Wirtschaftswachstum in den APRM Ländern zu stärken. Je stärker hier die Aussicht auf ein durch Good Governance induziertes endogenes Wirtschaftswachstum ist, d.h. je attraktiver der Quadrant I, desto größer ist für die regierende Elite der Anreiz, sich selbst zu binden. Hilft der APRM-Prozess hier, glaubhaft best practices zu identifizieren, ist insbesondere zu denken an einen verbesserten Marktzugang für die Produkte dieser Staaten, die Vergabe von Mikrokrediten oder die Berücksichtigung der APRM-Ergebnisse in internationalen Länderrankings, da diese wichtige Informationsanreize für multinationale Investitionen bereitstellen. ${ }^{64}$

(4) Die Überlegungen in Abschnitt 2 legen nahe, dass eine Selbstbindung im wohlverstandenen Eigeninteresse der herrschenden Elite liegt. So weit so gut. Wie die Praxis jedoch zeigt, nutzen keineswegs alle afrikanischen Staaten diesen Vorteil der Selbstbindung. Im Gegenteil. Rund die Hälfte aller Staaten hat es vorgezogen, sich dem APRM gar nicht erst anzuschließen. ${ }^{65}$ Hier können drei sich ergänzende Erklärungen entwickelt werden.

Erstens stellt der APRM ein (noch) sehr schwaches Bindungsinstrument dar. In Staaten, in denen die Glaubwürdigkeit der Elite aufgrund langjähriger Korruption, Bürgerkrieg und Misswirtschaft besonders gering ist, kann die mit dem APRM ver-

61 In Abbildung 2 entspräche dies der Strategie, den Payoff der Regierung in Quadrant I durch eine zusätzliche Zahlung X zu erhöhen. Gelingt dies, genügt bereits ein kleineres $\lambda$, um das Verhaltensgleichgewicht von Quadrant III zu Quadrant I zu überführen.

62 So bemerkt de Alex de Waal, FN 2, S. 475: "The rationale for the APR is that Africa should move away from donor-imposed conditionalities, which have been found to be ineffective, inefficient and burdensome, towards mutual accountability among development partners".

63 Um zu verhindern, dass die Vergabe von Entwicklungshilfe - vor allem aus Sicht der Länder mit einer vergleichsweise schlechten Governance-Performance - die Struktur eines Null-Summen-Spiels annimmt und damit Widerstände der möglichen Verlierer hervorruft, gilt es, in der Tat zusätzliche Gelder zur Unterstützung des APRM zu mobilisieren. Vgl. auch Alex de Waal, FN 2, S. 472.

64 Vgl. Ingo Pies / Cora Voigt, FN 22, S. 169, Fn. 20.

65 So haben es bis November 200726 NePAD-Staaten vorgezogen, dem APRM (noch) gar nicht beizutreten. In der Gruppe der APRM-Staaten hat wiederum nur eine Minderheit den Stand der reinen Absichtserklärung hinter sich gelassen und treibt den APRM-Prozess tatsächlich voran. 
bundene Sanktionswirkung 1 - aus Sicht der eigenen Bevölkerung! - schlichtweg zu schwach sein, um die Bevölkerung vom Reformwillen der Regierung zu überzeugen. In diesem Fall würde sich die Regierung durch eine - von der Bevölkerung nicht als glaubwürdig empfundene - Selbstbindung jedoch schlechter stellen: Sie würde nicht das endogene Wachstum in Quadrant I, sondern den im Vergleich zum Status quo in Quadrant III subjektiv noch schlechter bewerteten Quadrant II realisieren.

Zweitens hängt die Vorteilhaftigkeit einer Selbstbindung der Regierung von der Aussicht ab, in Quadrant I von endogenem Wirtschaftswachstum zu profitieren. Sieht die Regierung hier jedoch gar keine Aussicht auf Wirtschaftswachstum (etwa aufgrund fehlenden Marktzugangs, der AIDS-Pandemie oder gewalttätiger Konflikte), sinkt die erwartete Auszahlung in Quadrant I unter Umständen so stark, dass der Status quo in Quadrant III aus Sicht der Regierung als subjektiv vorteilhaft erscheint.

Der dritte Punkt ist politisch besonders sensibel. In diesem Fall unterminiert ausgerechnet das bestehende System von Entwicklungshilfe für einige Staaten den Anreiz, sich durch Mechanismen wie den APRM ernsthaft selbst zu binden. Wird, wie oftmals in der Vergangenheit, Entwicklungshilfe gerade jenen Ländern gegeben, denen es besonders schlecht geht, dann setzt dieses System für die herrschende Elite einen Anreiz, ihre eigene Bevölkerung gleichsam in Geiselhaft zu nehmen. ${ }^{66}$ Werden Entwicklungsgelder, von denen die Elite etwa durch Korruption profitiert, vor allem dann gezahlt, wenn es der Bevölkerung besonders schlecht geht, erfährt die herrschende Elite einen starken Anreiz, den Zustand dauerhafter Armut gar nicht $\mathrm{zu}$ ändern. ${ }^{67}$ Das Ausbeutungsgleichgewicht in Quadrant III wird dann so attraktiv, dass gar kein Regierungsinteresse mehr daran besteht, ein anderes Spiel zu spielen.

(5) Einige Beobachter argumentieren, dass das Gelingen des APRM davon abhänge, dass nicht nur einige, sondern alle NePAD-Mitglieder diesem Verfahren beitreten. Eine größtmögliche Inklusivität wird hier als Erfolgsbedingung des APRM gedeutet. ${ }^{68}$ Die hier entwickelten Überlegungen legen eine andere Lesart nahe. Nicht die Inklusivität des APRM, sondern gerade dessen Exklusivität ist eine zentrale Voraussetzung für eine möglichst hohe Bindungswirkun. Erklärt man den APRM für alle Staaten für verbindlich, dann wird sich als Standard der kleinste gemeinsame Nenner durchsetzen. Ist es dagegen einer Vorreitergruppe möglich, als Avantgarde zunächst eigene (höhere) Standards zu entwickeln, und führt diese Selbstbindung zu sichtbaren Erfolgen, dann steigt die Attraktivität des APRM lang-

66 Vgl. Ingo Pies / Cora Voigt, FN 22, S. 168.

67 Vgl. William Easterly, The White Man's Burden. Why the West's Efforts to Aid the Rest Have Done So Much Ill and So Little Good, New York 2006, insbesondere Kapitel 4 beginnend mit S. 112.

68 So kritisiert beispielsweise Cord Jakobeit, FN 3, S. 23, die Tatsache, dass es »die eigentlichen Problemfälle, wie z.B. Simbabwe, DR Kongo oder Elfenbeinküste, vorgezogen haben, sich dem APRM gar nicht erst anzuschließen. Letztlich wird die Entscheidung über Erfolg oder Scheitern des APRM davon abhängen, ob alle Staaten des Kontinents APRM beitreten.« 
fristig auch für andere Länder. Der Erfolg des APRM sollte daher nicht daran gemessen werden, ob alle NePAD-Länder den APRM in irgendeiner Weise anwenden, sondern ob der APRM in einigen, vielleicht anfangs nur wenigen Ländern tatsächlich eine spürbare Entwicklungsdynamik in Gang setzt bzw. unterstützt.

Eine differenzierte Anwendung des APRM eröffnet mithin die Möglichkeit, zu einer zentralen Gründungsidee der NePAD zurückzukehren. Während die einzige Bedingung für die Mitgliedschaft in der Afrikanischen Union in der geographischen Lage auf dem afrikanischen Kontinent besteht, lag eine entscheidende Neuerung der ursprünglichen NePAD-Konzeption darin, die Teilnahme in dieser Initiative von einer Reihe inhaltlicher Kriterien wie etwa Good Governance abhängig zu machen. ${ }^{69}$ Aus politischen Gründen wurde dieses Prinzip der Exklusivität Schritt für Schritt aufgehoben. Am Ende wurden sämtliche Länder - Demokratien wie Diktaturen - aufgenommen. ${ }^{70} \mathrm{NePADs}$ anfänglicher Anspruch, die Reformkräfte Afrikas zu bündeln, hat damit massiv an Glaubwürdigkeit verloren. Gerade die Freiwilligkeit des APRM könnte den reformorientierten Staaten eine alternative Arena eröffnen, eine Vorreiterrolle zu übernehmen und so eine Reform-Avantgarde innerhalb der NePAD-Strukturen zu bilden. Es bleibt abzuwarten, ob die inflationäre Ausweitung der APRM-Mitgliedschaft den eigentlichen Kerngedanken der Selbstbindung bereits unwiderruflich verwässert hat.

\section{Fazit und Ausblick}

Die New Partnership for Africa's Development und insbesondere der African Peer Review Mechanism haben hohe Erwartungen geweckt. Kritiker halten diese Erwartungen für unberechtigt. So wirft Ian Taylor einen äußerst skeptischen Blick auf NePAD. Er schreibt:

»The logic and modus operandi of neo-patrimonial rule and the dominance and nature of extractive economies in Africa - and their relationships with the international system - means that Nepad's strictures on good governance and democracy cannot be implemented without eroding the very nature of the post-colonial African state and undermining the positions of incumbent elites - an unlikely possibility.$^{71}$

Taylor hält aus diesem Grund den Ansatz von NePAD und des APRM für verfehlt. Vielmehr gelte es, zuerst die politischen Strukturen der neo-patrimonialen Herrschaft zu verändern, bevor ein Prozess wie NePAD überhaupt Erfolg haben könne. ${ }^{72}$

69 Vgl. Alex de Waal, FN 2, S. 469.

70 So bemerkt Ian Taylor (FN 6, S. 9): » The initial concept of NEPAD was that it was not going to be something any dictator could join to get the benefits, but a more narrow type of reformist club, this has now gradually shifted to become more inclusive."

71 Ian Taylor, FN 6, S. 2.

72 In diesem Sinne stellt Ian Taylor (FN 6) seinen Beitrag unter die (rhetorische) Titelfrage: »Can NEPAD succeed without prior political reform?«. 
Der vorliegende Beitrag hat entwickelt, dass sich mithilfe des Ansatzes der Wirtschaftsethik sozialer Dilemmata eine prononciert andere Sichtweise anleiten lässt. Aus dieser Perspektive lässt sich das Verhältnis von innerstaatlichen Reformen und kollektiven Initiativen wie dem APRM genau andersherum konzeptualisieren. Die Argumentation Taylors beruht auf einer reaktionsanalytischen Perspektive. Er betrachtet das neo-patrimoniale Politiksystem als ein gegebenes Spiel und folgert dann durchaus konsequent -, dass die herrschenden Elite gar kein Interesse daran hat, ihre Macht innerhalb dieses gegebenen Spiels einzuschränken. Die Wirtschaftsethik sozialer Dilemmata betrachtet NePAD hingegen aus einer interaktionsanalytischen Perspektive. Diese Sichtweise zeigt auf, dass das neo-patrimoniale Ausbeutungsspiel den Charakter einer sozialen Rationalfalle aufweist. Hier untergräbt die ungebundene Macht der Herrschenden Erwartungssicherheit und verhindert so Investitionen durch Unternehmen und Bevölkerung. Sowohl Bevölkerung als auch die Herrscherelite bleiben unter ihren Möglichkeiten. Insofern besteht auch für die regierende Elite ein Interesse, ein besseres Spiel zu spielen. Vor diesem Hintergrund lässt sich der African Peer Review Mechanism als ein Instrument der Selbstbindung interpretieren: als Mechanismus, um Reformanstrengungen glaubwürdig zu machen, Investitionen der eigenen Bevölkerung anzureizen und so das Unterentwicklungsgleichgewicht auf einen Pfad endogenen Wachstums zu überführen. Die von Taylor vorgeschlagene Reihenfolge wird in dieser Sichtweise folglich umgekehrt: Gerade die überstaatliche politische Selbstbindung durch den APRM eröffnet den Einstieg in ein produktiveres innerstaatliches Spiel, das nationale politische Reformen überhaupt erst anreizkompatibel macht.

So elegant das theoretische Potential des APRM als eine politische Selbstbindung rekonstruiert werden kann, so kritisch zu betrachten ist seine bisherige Ausgestaltung in der Praxis. In der tatsächlichen Umsetzung bietet der APRM (bis jetzt) nur einen schwachen Bindungsmechanismus. Harte Sanktionen fehlen hier völlig. Um daher überhaupt eine nennenswerte Wirkung entfalten zu können, bedarf es größtmöglicher Integrität und Glaubwürdigkeit des APRM. In dieser Hinsicht zeigt sich jedoch eine ganze Reihe von Problemen, angefangen mit der zweifelhaften Integrität einiger APRM-Mandatsträger über weitgehende Manipulationsmöglichkeiten der betroffenen Regierungen beim Erstellen der APRM-Berichte bis hin zu einer mangelnden Einbindung der Zivilgesellschaft vor Ort.

Es wäre allerdings verfehlt, den APRM daran zu messen, wie eine perfekte Selbstbindung in einer Theoriewelt aussähe. Entscheidend sind vielmehr die relevanten Alternativen. Hier gilt es einem zentralen Umstand Rechnung zu tragen: Aufgrund fehlender institutioneller Strukturen existieren effektive Bindungsmöglichkeiten in vielen Staaten Afrikas nur in begrenzter Form. Der Verfassungsstaat besteht oftmals nur auf dem Papier; die Bindungswirkung von Recht ist eingeschränkt; zivilgesellschaftliche Kontrollmechanismen sind unterentwickelt. Angesichts des Fehlens geeigneter innerstaatlicher Bindungsmöglichkeiten erweist sich der APRM zwar als ein durchaus mit eklatanten Mängeln behaftetes Instrument, aber letztlich als ein auch für die politikwissenschaftliche Forschung - interessanter Versuch, afrikanische Reformbemühungen zu stärken. Eine Unterstützung dieser Reformbemühung 
ist verschiedentlich denkbar: etwa durch Aufbau integrer Verfahrenskapazitäten für den APRM-Prozess; durch zusätzliche Wirtschaftshilfe, die best practices belohnt; durch die Öffnung von Märkten und die Bereitstellung von (Mikro-) Krediten, um die Attraktivität endogenen Wachstums zu fördern; und vor allem durch die Abkehr von der Praxis, Entwicklungshilfe dauerhaft jenen Regierungen zu geben, deren Bevölkerung es besonders schlecht geht.

In seiner ursprünglichen Konzeption ist der African Peer Review Mechanism daher als eine viel versprechende Innovation einzustufen. Tatsächlich hat er in einigen (wenigen) Ländern bereits substantiell dazu beigetragen, eine intensive Reformdebatte voranzutreiben. ${ }^{73}$ Trotzdem ist es angebracht, weder NePAD noch den APRM-Prozess mit Erwartungen zu überfrachten. Insbesondere wäre es dem Prozess abträglich, erwarten zu wollen, dass der APRM sofort und für sämtliche $\mathrm{Ne}$ PAD-Länder eine reformunterstützende Wirkung entfalten kann. Vielmehr haben die massiven Umsetzungsprobleme und insbesondere die Ausweitung der NePADInitiative auf praktisch gesamt Afrika das exklusive Bindungspotential des APRMMechanismus - vielleicht bereits unwiderruflich - verwässert. Um das Innovationspotential des APRM nicht vollständig zu untergraben, erscheint es daher sinnvoll, dass nun eine kleine, aber reformbereite Avantgarde-Gruppe mutig voranschreiten darf. Stellt sich der Erfolg ihrer Reformbemühungen erst einmal ein, mögen andere folgen.

\section{Zusammenfassung}

Der Beitrag analysiert das Potential politischer Selbstbindungen am Beispiel des African Peer Review Mechanism (APRM), einem wechselseitigen Informationsund Kontrollmechanismus im Kontext der Entwicklungsinitiative NePAD. Anhand des Ansatzes der Wirtschaftsethik sozialer Dilemmata wird diskutiert, welche politische Funktion der APRM systematisch erfüllen könnte. Das für Afrika charakteristische System neo-patrimonialer Herrschaft lässt sich als Unterentwicklungsgleichgewicht rekonstruieren. Die Überwindung dieser Rationalfalle bedarf einer wirksamen Selbstbindung der herrschenden Elite. Freilich wird der APRM diesem Anspruch in der bisherigen Umsetzung kaum gerecht. Allerdings gibt es angesichts schwacher Staatlichkeit oftmals keine stärkeren Bindungsalternativen. Vor diesem Hintergrund formuliert der Beitrag Gestaltungsempfehlungen zur Unterstützung der Selbstbindungswirkung des APRM.

\section{Abstract}

This paper discusses NePAD’s African Peer Review Mechanism (APRM) as an instructive case of political self-commitment. Recently, the APRM has been heavily criticized. Building on the theoretical approach of economic ethics and normative

73 Vgl. Ross Herbert, FN 11, S. 69. 
institutional analysis, the article seeks to delineate the systematic potential of political commitment strategies such as the APRM. The paper shows that the neo-patrimonial political system in many African states corresponds to a stable economic underdevelopment equilibrium. A simple model illustrates that the systematic logic of the APRM is to overcome this quasi-prisoners' dilemma through the self-commitment of political elites. In the field, however, the APRM so far fails to constitute a powerful self-commitment. Yet, in the face of limited statehood, most African states lack alternative and better mechanisms of political commitment. Against this background, the article spells out recommendations as to how to strengthen the APRM as a more credible commitment device.

Markus Beckmann, Putting Political Commitment Strategies into PerspectiveThe Case of NePAD and the African Peer Review Mechanism

\section{Welt Trends Papiere}

Sie möchten Forschungsarbeiten veröffentlichen?

Sie möchten in aktuelle Debatten streitbar eingreifen?

Sie möchten „klassische" Texte für die Lehre einsetzen?

Dies alles schnell, preiswert und für ein breites Publikum?

Wir bieten Ihnen dafür die WeltTrends Papiere!

1 Noch zu retten? Plädoyer für die EU-Verfassung

$2 \mid$ Deutsche EU-Ratspräsidentschaft Ende der Verfassungskrise?

$3 \mid$ Ansprüche? Eigentumsfrage Deutschland - Polen

4| Erfolgreich gescheitert! Die EU-Verfassungskrise

5| Deutsche Auslandseinsätze - Streitplatz um Militärmacht

6 Herausforderung Integration- Migration und Integration in Brandenburg

7| Großmachtpolitik oder Wahlagitation - Russlands Außenpolitik 2007/2008

Wenn Sie an der Publikation eines eigenen WT-Papieres interessiert sind, wenden Sie sich bitte an Frau Azadeh Zamirirad, M. A. (0331-9774540 oder welttrends@web.de).

Bestellungen: bestellung@welttrends.de oder ubpub@uni-potsdam.de Jedes WT-Papier nur $5 €$ www.welttrends.de 